\title{
Analytical study and numerical experiments for true and spurious eigensolutions of a circular cavity using the real-part dual BEM
}

\author{
S. R. Kuo, J. T. Chen ${ }^{* \dagger}$ and C. X. Huang \\ Department of Harbour and River Engineering, National Taiwan Ocean University, Keelung, Taiwan, R. O.C.
}

\begin{abstract}
SUMMARY
It has been found recently that the multiple reciprocity method (MRM) (Chen and Wong. Engng. Anal. Boundary Elements 1997; 20(1):25-33; Chen. Processings of the Fourth World Congress on Computational Mechanics, Onate E, Idelsohn SR (eds). Argentina, 1998; 106; Chen and Wong. J. Sound Vibration 1998; 217(1): 75-95.) or real-part BEM (Liou, Chen and Chen. J. Chinese Inst. Civil Hydraulics 1999; 11(2): 299-310 (in Chinese)). results in spurious eigenvalues for eigenproblems if only the singular (UT) or hypersingular $(L M)$ integral equation is used. In this paper, a circular cavity is considered as a demonstrative example for an analytical study. Based on the framework of the real-part dual BEM, the true and spurious eigenvalues can be separated by using singular value decomposition (SVD). To understand why spurious eigenvalues occur, analytical derivation by discretizing the circular boundary into a finite degree-of-freedom system is employed, resulting in circulants for influence matrices. Based on the properties of the circulants, we find that the singular integral equation of the real-part BEM for a circular domain results in spurious eigenvalues which are the zeros of the Bessel functions of the second kind, $Y_{n}(k \rho)$, while the hypersingular integral equation of the real-part BEM results in spurious eigenvalues which are the zeros of the derivative of the Bessel functions of the second kind, $Y_{n}^{\prime}(k \rho)$. It is found that spurious eigenvalues exist in the realpart BEM, and that they depend on the integral representation one uses (singular or hypersingular; single layer or double layer) no matter what the given types of boundary conditions for the interior problem are. Furthermore, spurious modes are proved to be trivial in the circular cavity through analytical derivations. Numerically, they appear to have the same nodal lines of the true modes after normalization with respect to a very small nonzero value. Two examples with a circular domain, including the Neumann and Dirichlet problems, are presented. The numerical results for true and spurious eigensolutions match very well with the theoretical prediction. Copyright (C) 2000 John Wiley \& Sons, Ltd.
\end{abstract}

KEY WORDS: real-part dual BEM; spurious eigenvalues and eigenmodes; singular value decomposition method; Bessel function

\footnotetext{
*Correspondence to: J. T. Chen, Department of Harbour and River Engineering, National Taiwan Ocean University, PO Box 7-59, Keelung, Taiwan, ROC

†E-mail: B0209@ntou66.ntou.edu.tw

Contract/grant sponsor: National Science Council; contract/grant number: NSC-88-2211-E-019-005, NSC-88-2815C-019-004-E
} 


\section{INTRODUCTION}

Eigenproblems are often encountered not only in vibration problems, but also in acoustic problems. Since exact solutions are sometimes not available, numerical methods are needed. Using the complex-valued boundary element method (BEM), the eigenvalues and eigenmodes can be determined [1]. To avoid the complex-valued computation, real-part formulation, imaginary-part formulation and multiple reciprocity method have been considered by some researchers. A simplified method using only the real-part or imaginary-part kernel was presented by DeMey [2]. However, only the first eigenvalue was studied in [2]. Also, Hutchinson replaced the complex-valued kernel by the real-part one to solve the plate and membrane vibration problems [3]. This novel method, using only the real-part BEM [4], was found to be equivalent to the multiple reciprocity method (MRM) if the zeroth-order fundamental solution is properly chosen [5]. In Reference [6], Chen and Wong found that MRM results in spurious eigensolutions for one-dimensional examples. Numerical experiments using real kernels were performed for two-dimensional cases [7]. Also, the relations among the conventional MRM, complete MRM, real-part BEM and complex-valued BEM were discussed in a keynote lecture by Chen [5]. It is very obvious that one advantage of using only the real-part kernels is that the real-valued computation is employed instead of complexvalued computation as used in the complex-valued BEM. Another gain is that lengthy derivation as required for the MRM can be avoided. However, two drawbacks of the real formulation have been found to be the occurrence of spurious eigenvalues $[6,7]$ and failure when it is applied to problems with a degenerate boundary [8]. To deal with these two problems, the framework of the real-part dual BEM was constructed to filter out spurious eigenvalues and to avoid nonunique solutions for problems with a degenerate boundary. As for the latter problem, the dual formulation is the key to solving problems with a degenerate boundary [8-13]. The reader can refer to a detailed review article on dual BEM, including 250 references, by Chen and Hong [14]. As for the former problem, the reason why spurious eigenvalues occur in the MRM or real-part BEM is the loss of constraints in the imaginary part, which was investigated in [15]. The fewer the constraint equations, the larger the solution space. It is not surprising that spurious eigenvalues occur in the MRM or real-part BEM formulation. By employing the dual MRM or real-part dual BEM, spurious eigenvalues can be filtered out by checking the residue between the singular and hypersingular equations, e.g. as in the two-dimensional cases studied in Reference [8]. A more efficient way, the SVD technique, was employed to filter out spurious modes for a rod [16], an Euler-Bernoulli beam [17], and a two-dimensional circular cavity [9]. The dual MRM and realpart dual BEM can be used to simultaneously solve the problems of spurious eigenvalues and nonunique solutions resulting from a degenerate boundary. However, the positions where spurious eigenvalues occur were not clearly understood in the past for two- and three-dimensional cases. Although spurious eigensolutions were found in one-dimensional cases (rod [16] and beam [17]) analytically and numerically, their existence for higher dimension cases were only discovered numerically but not analytically $[9,18]$. Predicting analytically where spurious eigenvalues will occur and how the spurious modes will behave was the goal of this study. That is to say, analytical solutions for both true and spurious eigenvalues are of interest. Also, numerical experiments using the real-part dual BEM were performed for comparison. Both direct and indirect methods will be examined here analytically.

In this paper, we employ the real-part dual BEM to solve the acoustic problems of a circular cavity. After assembling the dual equations, the singular value decomposition (SVD) technique [17] is employed to filter out spurious eigenvalues for two-dimensional cavities. Also, the boundary 
modes can be obtained directly by extracting the corresponding right singular vector of the right unitary matrix in SVD. Two examples for a circular domain, including the Dirichlet and Neumann problems, are employed to check the validity of the proposed method. The spurious eigensolutions are analytically predicted and are compared with those obtained by using the real-part dual BEM program, DUALREL. Finally, the true eigenvalues are derived analytically by approaching the discrete system as continuous using the analytical properties of circulants and are also compared with the numerical solutions obtained using the dual BEM program to check the validity of the present formulation.

\section{REVIEW OF THE REAL-PART DUAL INTEGRAL FORMULATION FOR A TWO-DIMENSIONAL ACOUSTIC CAVITY}

The governing equation for an acoustic cavity is the Helmholtz equation:

$$
\left(\nabla^{2}+k^{2}\right)\{u(\mathbf{x})\}=0, \quad \mathbf{x} \in D,
$$

where $\nabla^{2}$ is the Laplacian operator, $D$ is the domain of the cavity and $k$ is the wave number, which is the frequency over the speed of sound. The boundary conditions considered here are either of the Neumann or Dirichlet type.

Based on the complex-valued dual BEM [10, 19], the dual integral equations for the boundary points are

$$
\begin{array}{ll}
\pi u(\mathbf{x})=\mathrm{CPV} \int_{B} T(\mathbf{s}, \mathbf{x}) u(\mathbf{s}) \mathrm{d} B(\mathbf{s})-\mathrm{RPV} \int_{B} U(\mathbf{s}, \mathbf{x}) t(\mathbf{s}) \mathrm{d} B(\mathbf{s}), & \mathbf{x} \in B \\
\pi t(\mathbf{x})=\operatorname{HPV} \int_{B} M(\mathbf{s}, \mathbf{x}) u(\mathbf{s}) \mathrm{d} B(\mathbf{s})-\mathrm{CPV} \int_{B} L(\mathbf{s}, \mathbf{x}) t(\mathbf{s}) \mathrm{d} B(\mathbf{s}), & \mathbf{x} \in B
\end{array}
$$

in which CPV, RPV and HPV denote the Cauchy principal value, the Riemann principal value and Hadamard principal value; $t(\mathbf{s})=\partial u(\mathbf{s}) / \partial n_{s} ; B$ denotes the boundary enclosing $D$; and the four complex-valued kernels, $U, T, L$, and $M$ can be found in References [10,19]. Since the MRM has been proved to be no more than the real-part of the complex-valued formulation $[15,20]$, only real-part kernels will be adopted as follows:

$$
\begin{aligned}
& U_{\mathrm{R}}(\mathbf{s}, \mathbf{x})=\operatorname{Real}\{U(\mathbf{s}, \mathbf{x})\}=\frac{\pi Y_{0}^{(1)}(k r)}{2} \\
& T_{\mathrm{R}}(\mathbf{s}, \mathbf{x})=\operatorname{Real}\{T(\mathbf{s}, \mathbf{x})\}=\frac{k \pi}{2} Y_{1}^{(1)}(k r) \frac{y_{i} n_{i}}{r} \\
& L_{\mathrm{R}}(\mathbf{s}, \mathbf{x})=\operatorname{Real}\{L(\mathbf{s}, \mathbf{x})\}=\frac{-k \pi}{2} Y_{1}^{(1)}(k r) \frac{y_{i} \bar{n}_{i}}{r} \\
& M_{\mathrm{R}}(\mathbf{s}, \mathbf{x})=\operatorname{Real}\{M(\mathbf{s}, \mathbf{x})\}=\frac{k \pi}{2}\left\{-k \frac{Y_{2}^{(1)}(k r)}{r^{2}} y_{i} y_{j} n_{i} \bar{n}_{j}+\frac{Y_{1}^{(1)}(k r)}{r} n_{i} \bar{n}_{i}\right\}
\end{aligned}
$$

where $Y_{n}^{(1)}(k r)$ denotes the imaginary part of the $n$th order of the first-kind Hankel function $\left(H_{n}^{(1)}(k r)\right) ; r$ is the distance between the source point, $\mathbf{s}$, and the field point $\mathbf{x} ; n_{i}$ is the $i$ th 
component of the outnormal vector at $\mathbf{s}$; $\bar{n}_{i}$ is the $i$ th component of the outnormal vector at $\mathbf{x}$; and $y_{i} \equiv s_{i}-x_{i}$.

By employing the constant element scheme, Equations (1) and (2) can be discretized into the following linear algebraic equations:

$$
\begin{aligned}
& {\left[T_{\mathrm{R}}(k)-\pi I\right]\{u\}=\left[U_{\mathrm{R}}(k)\right]\{t\}} \\
& {\left[M_{\mathrm{R}}(k)\right]\{u\}=\left[L_{\mathrm{R}}(k)+\pi I\right]\{t\}}
\end{aligned}
$$

where $I$ is a unit matrix, and $\left[U_{\mathrm{R}}(k)\right],\left[T_{\mathrm{R}}(k)\right],\left[L_{\mathrm{R}}(k)\right]$ and $\left[M_{\mathrm{R}}(k)\right]$ are the influence matrices constructed by the $U_{\mathrm{R}}, T_{\mathrm{R}}, L_{\mathrm{R}}$ and $M_{\mathrm{R}}$ kernels. The detailed formulation can be found in Reference [7].

\section{DETECTION OF SPURIOUS EIGENVALUES USING THE REAL-PART DUAL BEM IN CONJUNCTION WITH THE SINGULAR VALUE DECOMPOSITION TECHNIQUE}

According to Equations (7) and (8), we can obtain the possible eigenvalues (true and spurious) independently for problems without degenerate boundaries. However, spurious roots are imbedded if the $U T$ equation (Equation (7)) or $L M$ equation (Equation (8)) is used alone. As mentioned by Kamiya et al. [20], the equation derived using the MRM is no more than that of the real part in the complex-valued formulation. Yeih et al. [15] extended the general proof for one and two dimensional problems and demonstrated it using a one-dimensional case. The loss of the imaginary part in the real-part BEM or MRM results in spurious roots. Their spurious eigenvalues occur at certain positions, which will be discussed in detail both analytically and numerically.

Since only the real part of the complex-valued kernels is of concern in the real-part BEM or MRM, one approach to obtaining enough constraints for the eigenequation instead of the imaginary part of the complex-valued formulation is to derive the secondary field equation by taking normal derivative of the primary field in real-part BEM or conventional MRM [21] with respect to the field point. This method results in the hypersingular formulation for the real-part BEM or MRM. For demonstration purposes, we will deal with the Neumann problem. Therefore, Equations (7) and (8) reduce to

$$
\begin{aligned}
{\left[\bar{T}_{\mathrm{R}}(k)\right]_{N \times N}\{u\}_{N \times 1} } & =\{0\} \\
{\left[M_{\mathrm{R}}(k)\right]_{N \times N}\{u\}_{N \times 1} } & =\{0\}
\end{aligned}
$$

where $N$ is the number of boundary elements. To find spurious eigenvalues using the SVD technique, we can merge the two matrices in Equations (9) and (10) together to obtain

$$
[J(k)]_{2 N \times N}\{u\}_{N \times 1}=\{0\}
$$

where the $[J(k)]$ matrix is assembled from the $[\bar{T}]$ and $[M]$ matrices as shown below

$$
[J(k)]_{2 N \times N}=\left[\begin{array}{c}
\bar{T}_{\mathrm{R}}(k) \\
M_{\mathrm{R}}(k)
\end{array}\right]
$$

Even though the $[J(k)]$ matrix has dependent rows resulting from the degenerate boundary, the SVD technique can still be employed to find all the true eigenvalues since a sufficient number 
of constraints is imbedded in the overdeterminate matrix, $[J(k)]$. As for the true eigenvalues, the rank of the $[J(k)]$ matrix with dimension $2 N \times N$ must at most be $N-1$ to obtain a non-trivial solution. As for the spurious eigenvalues, the rank must be $N$ to obtain a trivial solution. Based on this criterion, the SVD technique can be employed to detect the true eigenvalues by checking whether or not the first minimum singular values, $\sigma_{1}$, are zeros. Since discretization creates errors, very small values for $\sigma_{1}$, but not exactly zeros, will be obtained when $k$ is near the critical wave number. In order to avoid the need to determine the threshold for the zero numerically, a value of $\sigma_{1}$ closer to zero must be obtained using a smaller increment near the critical wave number, $k$. Such a value is confirmed to be a true eigenvalue.

Employing the SVD technique, we have

$$
[J(k)]_{2 N \times N}=[U]_{2 N \times 2 N}[\Sigma]_{2 N \times N}[V]_{N \times N}^{*}
$$

where $[U]$ is a left unitary matrix constructed by the left singular vectors, and $[\Sigma]$ is a diagonal matrix which has singular values $\sigma_{1}, \sigma_{2}, \ldots$, and $\sigma_{N}$ allocated in a diagonal line as

$$
[\Sigma]=\left[\begin{array}{ccc}
\sigma_{N} & \cdots & 0 \\
\vdots & \ddots & \vdots \\
0 & \cdots & \sigma_{1} \\
\vdots & \ddots & \vdots \\
0 & \cdots & 0
\end{array}\right]
$$

in which $\sigma_{N} \geqslant \sigma_{N-1} \cdots \geqslant \sigma_{1}$ and $[V]^{*}$ is the complex conjugate transpose of a right unitary matrix constructed by the right singular vectors. As we can see in Equation (14), there exist at most $N$ non-zero singular values.

Since we have employed the SVD technique to filter out spurious eigenvalues, we can obtain the boundary mode by extracting the right singular vector in the right unitary matrix of SVD corresponding to the near zero or zero singular value.

According to the properties of SVD [23,24], we have

$$
[J] \mathbf{v}_{p}=\sigma_{p} \mathbf{u}_{p}, \quad p=1,2,3, \ldots, n
$$

If the $q$ th singular value, $\sigma_{q}$, is zero, then we have the following equation immediately from Equation (15):

$$
[J] \mathbf{v}_{q}=0 \mathbf{u}_{q}=\mathbf{0}, \quad q=1,2,3, \ldots, Q
$$

where the root is generally assumed to be a $Q$-tuple root. Based on Equation (16), the non-trivial boundary mode is found to be the right singular vector, $\mathbf{v}_{q}$, in the right unitary matrix.

\section{ANALYTICAL STUDY OF TRUE AND SPURIOUS EIGENSOLUTIONS USING DIRECT AND INDIRECT METHODS}

Here, we will demonstrate analytical derivation for the true and spurious eigensolutions of a circular cavity using both indirect and direct methods. 


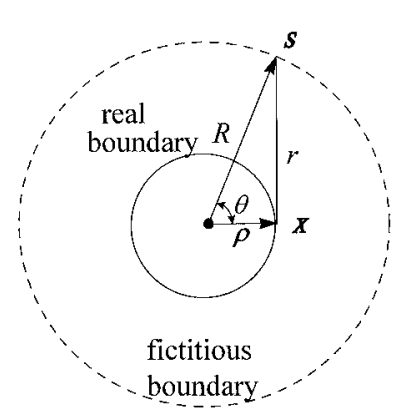

(a)

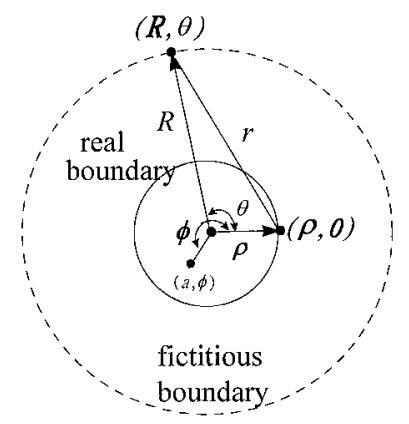

(b)

Figure 1. (a) The definitions of $\rho, \theta$, and $R$; (b) the definitions of $\rho, \theta, \phi, a$ and $R$.

Indirect formulation: Based on the degenerate (separable) properties for the $U_{\mathrm{R}}$ kernels, the real-part $U_{\mathrm{R}}$ kernel can be expanded into

$$
U_{\mathrm{R}}(\mathbf{x}, \mathbf{s})=\frac{\pi}{2} Y_{0}(k r)=\frac{\pi}{2} Y_{0}\left(k \sqrt{R^{2}+\rho^{2}-2 R \rho \cos \theta}\right)
$$

where $\rho, r, R$ and $\theta$ are shown in Figure 1(a). Since $\mathbf{x}$ and $\mathbf{s}$ are on circular boundaries with radius $\rho$ and $R$, respectively, $U(\mathbf{x}, \mathbf{s})$ can be expanded into

$$
U_{\mathrm{R}}(\mathbf{x}, \mathbf{s})=U(\theta)=\sum_{m=-\infty}^{\infty} \frac{\pi}{2} Y_{m}(k R) J_{m}(k \rho) \cos (m \theta), R>\rho
$$

where the source and field points in the two-point function $U_{\mathrm{R}}(x, s)$ are separated and $J_{m}(k \rho)$ is the $m$ th order Bessel function of the first kind. By superimposing $2 N$ constant source distribution $\{\bar{t}\}$ along the fictitious boundary with radius $R$ and collocating the $2 N$ points on the real boundary with radius $\rho$, we have

$$
\{u\}=[A]\{\bar{t}\}=\left[\begin{array}{cccccc}
a_{0} & a_{1} & a_{2} & \cdots & a_{2 N-2} & a_{2 N-1} \\
a_{2 N-1} & a_{0} & a_{1} & \cdots & a_{2 N-3} & a_{2 N-2} \\
a_{2 N-2} & a_{2 N-1} & a_{0} & \cdots & a_{2 N-4} & a_{2 N-3} \\
\vdots & \vdots & \vdots & \ddots & \vdots & \vdots \\
a_{1} & a_{2} & a_{3} & \cdots & a_{2 N-1} & a_{0}
\end{array}\right]\left\{\begin{array}{c}
\bar{t}_{0} \\
\bar{t}_{1} \\
\bar{t}_{2} \\
\vdots \\
\bar{t}_{2 N-1}
\end{array}\right\}=\{0\}
$$

for the Dirichlet problem, where $\bar{t}_{j}$ is the fictitious density of single layer potential distributed on the circular boundary with radius $R$, and $[A]$ is the influence matrix with the elements shown below:

$$
a_{m}=\int_{(m-1 / 2) \Delta \theta}^{(m+1 / 2) \Delta \theta} U(\theta) R \mathrm{~d} \theta \approx U\left(\theta_{m}\right) R \Delta \theta, \quad m=0,1,2, \ldots, 2 N-1
$$

where $\Delta \theta=2 \pi / 2 N$ and $\theta_{m}=m \Delta \theta$.

Matrix $[A]$ in Equation (19) is found to be in circulant form since rotation symmetry for the influence coefficients exists. By introducing the following bases for the circulants: $I, C_{2 N}^{1}, C_{2 N}^{2}, \ldots, C_{2 N}^{2 N-1}$, we can expand $[A]$ into [22]

$$
[A]=a_{0} I+a_{1} C_{2 N}^{1}+a_{2} C_{2 N}^{2}+\cdots+a_{2 N-1} C_{2 N}^{2 N-1}
$$


where

$$
C_{2 N}=\left[\begin{array}{cccccc}
0 & 1 & 0 & \cdots & 0 & 0 \\
0 & 0 & 1 & \cdots & 0 & 0 \\
\vdots & \vdots & \vdots & \ddots & \vdots & \vdots \\
0 & 0 & 0 & \cdots & 0 & 1 \\
1 & 0 & 0 & \cdots & 0 & 0
\end{array}\right]_{2 N \times 2 N}
$$

Based on the similar properties of the matrices of $[A]$ and $\left[C_{2 N}\right]$, we have

$$
\lambda_{\ell}=a_{0}+a_{1} \alpha_{\ell}+a_{2} \alpha_{\ell}^{2}+\cdots+a_{2 N-1} \alpha_{\ell}^{2 N-1}, \quad \ell=0, \pm 1, \pm 2, \ldots, \pm(N-1), N
$$

where $\lambda_{\ell}$ and $\alpha_{\ell}$ are the eigenvalues for $[A]$ and $\left[C_{2 N}\right]$, respectively. It is easily found that the eigenvalues and eigenvectors for the circulants $\left[C_{2 N}\right]$ are the roots for $\alpha^{2 N}=1$ as shown below:

$$
\alpha_{n}=\mathrm{e}^{\mathrm{i} 2 \pi n / 2 N}, \quad n=0, \pm 1, \pm 2, \ldots, \pm(N-1), N \text { or } n=0,1,2, \ldots, 2 N-1
$$

$$
\{\phi\}_{n}=\left\{\begin{array}{c}
1 \\
\alpha_{n} \\
\alpha_{n}^{2} \\
\alpha_{n}^{3} \\
\vdots \\
\alpha_{n}^{2 N-1}
\end{array}\right\}
$$

respectively.

Substituting Equation (24) into Equation (23), we have

$$
\lambda_{\ell}=\sum_{m=0}^{2 N-1} a_{m} \alpha_{\ell}^{m}=\sum_{m=0}^{2 N-1} a_{m} \mathrm{e}^{\mathrm{i} 2 \pi / 2 N m \ell} \quad \ell=0, \pm 1, \pm 2, \ldots, \pm(N-1), N
$$

According to the definition for $a_{m}$ in Equation (20), we have

$$
a_{m}=a_{2 N-m}, \quad m=0,1,2, \ldots, 2 N-1
$$

Substituting Equation (27) into Equation (26), we have

$$
\begin{aligned}
\lambda_{\ell} & =a_{0}+(-1)^{\ell} a_{N}+\sum_{m=1}^{N-1}\left(\alpha_{\ell}^{m}+\alpha_{\ell}^{2 N-m}\right) a_{m} \\
& =\sum_{m=0}^{2 N-1} \cos (m \ell \Delta \theta) a_{m}
\end{aligned}
$$


Substituting $a_{m}$ in Equation (20) into Equation (28), we have

$$
\lambda_{\ell} \approx \sum_{m=0}^{2 N-1} \cos (m \ell \Delta \theta) U(m \Delta \theta) R \Delta \theta=\int_{0}^{2 \pi} \cos (\ell \theta) U(\theta) R \mathrm{~d} \theta
$$

as $N$ approaches infinity. Equation (29) reduces to

$$
\begin{aligned}
\lambda_{\ell} & =\int_{0}^{2 \pi} \cos (\ell \theta) \sum_{m=-\infty}^{\infty} \frac{\pi}{2} Y_{m}(k R) J_{m}(k \rho) \cos m \theta R \mathrm{~d} \theta \\
& =\pi^{2} R Y_{\ell}(k R) J_{\ell}(k \rho)
\end{aligned}
$$

Since the wave number $k$ is embedded in each element of the $[A]$ matrix, the eigenvalues for $[A]$ are also functions of $k$. Finding the eigenvalues for the Helmholtz equation or finding the zeros for the determinant of $[A]$ is equal to finding the zeros for multiplication of all the eigenvalues of $[A]$. Based on the following equation:

$$
\operatorname{det}[A]=\lambda_{0} \lambda_{N}\left(\lambda_{1} \lambda_{2} \cdots \lambda_{N-1}\right)\left(\lambda_{-1} \lambda_{-2} \cdots \lambda_{-(N-1)}\right)
$$

the possible eigenvalues (true or spurious) occur at

$$
Y_{\ell}(k R) J_{\ell}(k \rho)=0, \quad \ell=0, \pm 1, \pm 2, \ldots, \pm(N-1), N
$$

Since the alternating properties for the Bessel function can be obtained, i.e.

$$
\begin{array}{ll}
Y_{-\ell}(k R)=(-1)^{\ell} Y_{\ell}(k R), & \ell \in \mathbf{N} \\
J_{-\ell}(k R)=(-1)^{\ell} J_{\ell}(k R), & \ell \in \mathbf{N}
\end{array}
$$

Equation (31) can be reduced to

$$
\operatorname{det}[A]=\lambda_{0}\left(\lambda_{1} \lambda_{2} \cdots \lambda_{N-1}\right)^{2} \lambda_{N}
$$

The square term in Equation (35) implies that double roots occur for $\lambda_{\ell}$ when $\ell=1,2, \ldots, N-1$. In order to verify that either $J_{\ell}(k \rho)=0$ or $Y_{\ell}(k R)=0$ is a true eigenequation, another approach is needed and is described in the following.

Based on the method of double-layer potential, we have the kernel $U_{\mathrm{R}}^{*}(\mathbf{x}, \mathbf{s})$ shown below:

$$
U_{\mathrm{R}}^{*}(\mathbf{x}, \mathbf{s})=\frac{\pi}{2} \frac{\partial Y_{0}(k r)}{\partial R}=\frac{\pi}{2} \frac{\partial Y_{0}\left(k \sqrt{R^{2}+\rho^{2}-2 R \rho \cos \theta}\right)}{\partial R}
$$

Similarly, $U_{\mathrm{R}}^{*}(\mathbf{x}, \mathbf{s})$ can be separated into

$$
U_{\mathrm{R}}^{*}(\mathbf{x}, \mathbf{s})=U_{\mathrm{R}}^{*}(\theta)=\sum_{m=-\infty}^{\infty} \frac{\pi k}{2} Y_{m}^{\prime}(k R) J_{m}(k \rho) \cos (m \theta), \quad R>\rho
$$

In a similar way of using circulants, we have the possible eigenequations as follows:

$$
Y_{\ell}^{\prime}(k R) J_{\ell}(k \rho)=0, \quad \ell=0, \pm 1, \pm 2, \ldots, \pm(N-1), N
$$


Comparing Equation (32) with Equation (38), we can easily find that $J_{m}(k \rho)=0$ is the true eigenequation. The spurious eigenequations are $Y_{m}(k R)=0$ and $Y_{m}^{\prime}(k R)=0$ if single- and doublelayer potentials are employed, respectively.

Direct formulation: In the limiting case of $R \rightarrow \rho$ for comparison with the direct method, Equation (32) reduces to

$$
Y_{\ell}(k \rho) J_{\ell}(k \rho)=0
$$

Similarly, we have

$$
\lambda_{\ell}=\int_{0}^{2 \pi} U_{\mathrm{R}}^{*}(\theta) \cos (\ell \theta) \rho \mathrm{d} \theta
$$

if the double-layer potentials are superimposed. Since $U_{\mathrm{R}}^{*}(\theta)$ is singular for the case of $R=\rho$ and $\theta=0^{\circ}$, the regularization technique is employed as follows:

$$
\begin{aligned}
U_{\mathrm{R}}^{*}(\theta) & =\sum_{m=-\infty}^{\infty} \frac{\pi k}{2} Y_{m}^{\prime}(k \rho) J_{m}(k \rho) \cos m \theta \\
& =\frac{\pi k}{4} \sum_{m=-\infty}^{\infty}\left(Y_{m}^{\prime} J_{m}+Y_{m} J_{m}^{\prime}\right) \cos m \theta+\frac{\pi k}{4} \sum_{m=-\infty}^{\infty}\left(Y_{m}^{\prime} J_{m}-Y_{m} J_{m}^{\prime}\right) \cos m \theta \\
& =U_{\mathrm{r}}^{*}(\theta)+\frac{1}{\rho} \delta(\theta)
\end{aligned}
$$

using addition and subtraction techniques, where $U_{\mathrm{r}}^{*}(\theta)$ is a regular function and the singular function is decomposed, after using the Wronskian $W$, for $J_{m}$ and $Y_{m}$,

$$
W\left(J_{m}, Y_{m}\right)=Y_{m}^{\prime} J_{m}-Y_{m} J_{m}^{\prime}=\frac{2}{\pi k \rho}
$$

and

$$
\sum_{m=-\infty}^{\infty} \cos m \theta=2 \delta(\theta)
$$

in which $\delta(\theta)$ is the Dirac-Delta function. Therefore,

$$
\begin{aligned}
\lambda_{\ell} & =\int_{0}^{2 \pi} U_{\mathrm{R}}^{*}(\theta) \cos (\ell \theta) \rho \mathrm{d} \theta \\
& =\pi+\int_{0}^{2 \pi} U_{\mathrm{r}}^{*}(\theta) \cos (\ell \theta) \rho \mathrm{d} \theta \\
& =\frac{\pi^{2} k \rho}{2}\left[Y_{\ell}^{\prime}(k \rho) J_{\ell}(k \rho)+Y_{\ell}(k \rho) J_{\ell}^{\prime}(k \rho)\right]+\pi \\
& =\pi^{2} k \rho Y_{\ell}^{\prime}(k \rho) J_{\ell}(k \rho)
\end{aligned}
$$


after using Equation (41) such that the jump source can be separated. Comparing Equations (39) and (44), we find that the true eigenvalue occurs at the zeros for $J_{\ell}(k \rho)=0$ while the spurious eigenvalues occur at the zeros for $Y_{\ell}(k \rho)=0$ or $Y_{\ell}^{\prime}(k \rho)=0$ if the singular equation $(U, T)$ and hypersingular equation $(L, M)$ are employed, respectively.

Based on the same algorithm, we can summarize the corresponding results in the following eigenequations:

Spurious eigenequations for both the Dirichlet and Neumann problems:

$$
\begin{aligned}
& Y_{\ell}(k R)=0, R \geqslant \rho \text { using the indirect method (single-layer, } U_{\mathrm{R}}(x, s), T_{\mathrm{R}}(x, s) \text { ) } \\
& Y_{\ell}^{\prime}(k R)=0, R \geqslant \rho \text { using the indirect method (double-layer, } U_{\mathrm{R}}^{*}(x, s), T_{\mathrm{R}}^{*}(x, s) \text { ) } \\
& Y_{\ell}(k \rho)=0 \text { using the direct method (UT equation, } U_{\mathrm{R}}(s, x), T_{\mathrm{R}}(s, x) \text { ) } \\
& \left.Y_{\ell}^{\prime}(k \rho)=0 \text { using the direct method (LMequation, } L_{\mathrm{R}}(s, x), M_{\mathrm{R}}(s, x)\right)
\end{aligned}
$$

where $k R$ or $k \rho$ using the indirect methods depend on the boundary where singularities are distributed, and the relations among the eight kernels $\left(U_{\mathrm{R}}(s, x), T_{\mathrm{R}}(s, x), L_{\mathrm{R}}(s, x), M_{\mathrm{R}}(s, x), U_{\mathrm{R}}(x, s)\right.$, $\left.U_{\mathrm{R}}^{*}(x, s), T_{\mathrm{R}}(x, s), T_{\mathrm{R}}^{*}(x, s)\right)$ between the direct and indirect methods in Equations (45)-(48) are shown in Table I.

True eigenequations for both the direct and indirect methods are:

$$
\begin{gathered}
J_{\ell}(k \rho)=0 \text { for the Dirichlet problem } \\
J_{\ell}^{\prime}(k \rho)=0 \text { for the Neumann problem }
\end{gathered}
$$

The above results are summaized in Table II. After determining the eigenvalues, the boundary modes are our concern. It is interesting to find that both the true and spurious boundary modes are found to be the same as shown in Equation (25) since matrix $[A]$ is similar to the circulant in Equation (22).

\section{ANALYTICAL DERIVATIONS FOR TRUE AND SPURIOUS INTERIOR MODES}

For the case of the Dirichlet problem, we have

$$
\left.u(r, \theta)\right|_{r=\rho}=0, \quad 0<\theta<2 \pi
$$

The boundary eigenvectors using the methods of single or double layer are the same as the eigenvectors in Equation (25) for the $\left[C_{2 N}\right]$ matrix since $[A]$ and $\left[C_{2 N}\right]$ are similar to each other. The $U_{\mathrm{R}}(\mathbf{x}, \mathbf{s})$ kernel can be expressed as

$$
U_{\mathrm{R}}(\mathbf{x}, \mathbf{s})=\sum_{m=-\infty}^{\infty} \frac{\pi}{2} J_{m}(k a) Y_{m}(k R) \cos (m(\theta-\phi))=U_{a}(\theta-\phi)
$$


Table I. Relations of the kernels in the direct and indirect methods using the dual model where $U_{\mathrm{R}}(s, x)=\pi Y_{0}(k r) / 2$ for the Helmholtz equation.

\begin{tabular}{|c|c|c|c|c|c|c|}
\hline $\begin{array}{l}\text { Kernel } \\
\text { function } \\
K(s, x) \\
\text { direct } \\
\text { method }\end{array}$ & $U_{\mathrm{R}}(s, x)$ & $T_{\mathrm{R}}(s, x)$ & $L_{\mathrm{R}}(s, x)$ & $M_{\mathrm{R}}(s, x)$ & $L_{\mathrm{R}}^{t}(s, x)$ & $M_{\mathrm{R}}^{t}(s, x)$ \\
\hline $\begin{array}{l}\text { Kernel } \\
\text { function } \\
K(x, s) \\
\text { indirect } \\
\text { method }\end{array}$ & $U_{\mathrm{R}}(x, s)$ & $U_{\mathrm{R}}^{*}(x, s)$ & $T_{\mathrm{R}}(x, s)$ & $T_{\mathrm{R}}^{*}(x, s)$ & $T_{\mathrm{R}}^{t}(x, s)$ & $T_{\mathrm{R}}^{* t}(x, s)$ \\
\hline Singularity 1D & $O(r)$ & $O(1)$ & $O(1)$ & $O(\delta(r))$ & $O(1)$ & $O(\delta(r))$ \\
\hline Singularity 2D & $O(\ln (r))$ & $O(1 / r)$ & $O(1 / r)$ & $O\left(1 / r^{2}\right)$ & $O(1 / r)$ & $O\left(1 / r^{2}\right)$ \\
\hline Singularity 3D & $O(1 / r)$ & $O\left(1 / r^{2}\right)$ & $O\left(1 / r^{2}\right)$ & $O\left(1 / r^{3}\right)$ & $O\left(1 / r^{2}\right)$ & $O\left(1 / r^{3}\right)$ \\
\hline $\begin{array}{l}\text { Density } \\
\text { function } \\
\mu(s)\end{array}$ & $-t$ & $u$ & $-t$ & $u$ & $-t$ & $u$ \\
\hline $\begin{array}{l}\text { Potential } \\
\text { type } \\
\int K(s, x) \mu(s) \mathrm{d} s\end{array}$ & $\begin{array}{l}\text { Single } \\
\text { layer }\end{array}$ & $\begin{array}{l}\text { Double } \\
\text { layer }\end{array}$ & $\begin{array}{c}\text { Normal } \\
\text { derivative } \\
\text { of single } \\
\text { layer } \\
\text { potential }\end{array}$ & $\begin{array}{c}\text { Normal } \\
\text { derivative } \\
\text { of double } \\
\text { layer } \\
\text { potential }\end{array}$ & $\begin{array}{c}\text { Tangent } \\
\text { derivative } \\
\text { of single } \\
\text { layer } \\
\text { potential }\end{array}$ & $\begin{array}{c}\text { Tangent } \\
\text { derivative } \\
\text { of double } \\
\text { layer } \\
\text { potential }\end{array}$ \\
\hline $\begin{array}{l}\text { Continuity } \\
\text { across } \\
\text { boundary }\end{array}$ & $\begin{array}{l}\text { Conti- } \\
\text { nuous }\end{array}$ & $\begin{array}{l}\text { Disconti- } \\
\text { nuous }\end{array}$ & $\begin{array}{l}\text { Disconti- } \\
\text { nuous }\end{array}$ & $\begin{array}{l}\text { Pseudo- } \\
\text { conti- } \\
\text { nuous }\end{array}$ & $\begin{array}{l}\text { Conti- } \\
\text { nuous }\end{array}$ & $\begin{array}{l}\text { Disconti- } \\
\text { nuous }\end{array}$ \\
\hline $\begin{array}{l}\text { Principal } \\
\text { value } \\
\text { sense }\end{array}$ & RPV & $\mathrm{CPV}$ & $\mathrm{CPV}$ & HPV & $\mathrm{CPV}$ & $\mathrm{HPV}$ \\
\hline
\end{tabular}

where the interior point for $\mathbf{x}=(a, \phi)$ in terms of the polar coordinate is shown in Figure 1(b). Based on the single-layer potential solution, we have

$$
\begin{aligned}
u_{n}(a, \phi) & =\sum_{\ell=0}^{2 N-1} U_{a}(\ell \Delta \theta-\phi) \bar{t}_{\ell n} R \Delta \theta=\sum_{\ell=0}^{2 N-1} U_{a}(\ell \Delta \theta-\phi) \cos (\ell n \Delta \theta) R \Delta \theta \\
& =\int_{0}^{2 \pi} U_{a}(\theta-\phi) \cos (n \theta) R \mathrm{~d} \theta \\
& =\int_{0}^{2 \pi} \sum_{m=-\infty}^{\infty} \frac{\pi}{2} J_{m}(k a) Y_{m}(k R) \cos (m(\theta-\phi)) \cos (n \theta) R \mathrm{~d} \theta \\
& =\pi^{2} R J_{n}(k a) Y_{n}(k R) \cos (n \phi), \quad 0<a<\rho, \quad 0<\phi<2 \pi
\end{aligned}
$$


Table II. The true and spurious eigenequations for a circular cavity under Dirichlet and Neumann boundary conditions using different formulations where $n=0,1,2,3, \ldots$.

\begin{tabular}{|c|c|c|c|c|}
\hline \multirow[b]{2}{*}{ Direct methods } & \multicolumn{2}{|c|}{ Interior Dirichlet problem } & \multicolumn{2}{|c|}{ Interior Neumann problem } \\
\hline & True eigenequation & Spurious eigenequation & True eigenequation & Spurious eigenequation \\
\hline$U(\mathbf{s}, \mathbf{x}), T(\mathbf{s}, \mathbf{x})$ & $J_{n}(k \rho)=0$ & $x$ & $J_{n}^{\prime}(k \rho)=0$ & $x$ \\
\hline$L(\mathbf{s}, \mathbf{x}), M(\mathbf{s}, \mathbf{x})$ & $J_{n}(k \rho)=0$ & $x$ & $J_{n}^{\prime}(k \rho)=0$ & $x$ \\
\hline$U_{R}(\mathbf{s}, \mathbf{x}), T_{R}(\mathbf{s}, \mathbf{x})$ & $J_{n}(k \rho)=0$ & $Y_{n}(k \rho)=0$ & $J_{n}^{\prime}(k \rho)=0$ & $Y_{n}(k \rho)=0$ \\
\hline$L_{R}(\mathbf{s}, \mathbf{x}), M_{R}(\mathbf{s}, \mathbf{x})$ & $J_{n}(k \rho)=0$ & $Y_{n}^{\prime}(k \rho)=0$ & $J_{n}^{\prime}(k \rho)=0$ & $Y_{n}^{\prime}(k \rho)=0$ \\
\hline $\begin{array}{l}\text { Indirect } \\
\text { methods }\end{array}$ & $\begin{array}{c}\text { True } \\
\text { eigenequation }\end{array}$ & $\begin{array}{c}\text { Spurious } \\
\text { eigenequation }\end{array}$ & $\begin{array}{c}\text { True } \\
\text { eigenequation }\end{array}$ & $\begin{array}{c}\text { Spurious } \\
\text { eigenequation }\end{array}$ \\
\hline$U(\mathbf{x}, \mathbf{s}), U^{*}(\mathbf{x}, \mathbf{s})$ & $J_{n}(k \rho)=0$ & $x$ & $J_{n}^{\prime}(k \rho)=0$ & $x$ \\
\hline$T(\mathbf{x}, \mathbf{s}), T^{*}(\mathbf{x}, \mathbf{s})$ & $J_{n}(k \rho)=0$ & $x$ & $J_{n}^{\prime}(k \rho)=0$ & $x$ \\
\hline$U_{R}(\mathbf{x}, \mathbf{s}), T_{R}(\mathbf{x}, \mathbf{s})$ & $J_{n}(k \rho)=0$ & $Y_{n}(k R)=0, R \geqslant \rho$ & $J_{n}^{\prime}(k \rho)=0$ & $Y_{n}(k R)=0, R \geqslant \rho$ \\
\hline$U_{R}^{*}(\mathbf{x}, \mathbf{s}), T_{R}^{*}(\mathbf{x}, \mathbf{s})$ & $J_{n}(k \rho)=0$ & $Y_{n}^{\prime}(k R)=0, R \geqslant \rho$ & $J_{n}^{\prime}(k \rho)=0$ & $Y_{n}^{\prime}(k R)=0, R \geqslant \rho$ \\
\hline
\end{tabular}

after considering the real part for the $\ell$ th component, $\bar{t}_{\ell n}$, in the eigenvector $\{\phi\}_{n}$ of Equation (25). If the sine part of the eigenvector in Equation (25) is chosen, the interior mode becomes

$$
u_{n}(a, \phi)=\pi^{2} R J_{n}(k a) Y_{n}(k R) \sin (n \phi), \quad 0<a<\rho, \quad 0<\phi<2 \pi
$$

Equation (54) shows the interior modes when $k$ represents true eigenvalues for the Dirichlet problems which satisfy $J_{n}(k \rho)=0$. Also, Equation (54) shows the spurious modes when $k$ represents spurious eigenvalues which satisfy $Y_{n}(k R)=0$. From a theoretical standpoint, it is interesting to find that the spurious modes are trivial since the term $Y_{n}(k R)=0$ is imbedded in Equation (54). However, the spurious modes obtained numerically can be normalized as

$$
\bar{u}_{n}(a, \phi)=\frac{u_{n}(a, \phi)}{\pi^{2} R Y_{n}(k R)}=J_{n}(k a) \cos (n \phi)
$$

where $\bar{u}_{n}(a, \phi)$ is a normalized mode. Equation (55) indicates that the nodal lines for true and spurious modes are the same after normalization as shown in Equations (53) and (55) if $Y_{n}(k R)$ approaches zero but is not exactly zero numerically. Nevertheless, true and spurious modes are quite different since the values of $k$ are not the same (one is true and the other is spurious) [25].

Similarly, the kernel for $U_{\mathrm{R}}^{*}(\mathbf{x}, \mathbf{s})$ can be expressed as

$$
U_{\mathrm{R}}^{*}(\mathbf{x}, \mathbf{s})=\sum_{m=-\infty}^{\infty} \frac{k \pi}{2} J_{m}(k a) Y_{m}^{\prime}(k R) \cos (m(\theta-\phi))=U_{a}^{*}(\theta-\phi)
$$


Table III. True and spurious systems for the Dirichlet problem using the real-part UT and LM BEMs.

\begin{tabular}{|c|c|c|c|c|}
\hline & \multicolumn{2}{|c|}{ Eigenequation } & $\begin{array}{l}\text { Eigenmode } \\
\text { (boundary) }\end{array}$ & $\begin{array}{l}\text { Eigenmode(interior): } u_{n}(a, \phi) \text { (unnormalized) } \\
\text { Eigenmode(interior): } \bar{u}_{n}(a, \phi) \text { (normalized) }\end{array}$ \\
\hline \multirow[t]{2}{*}{ UT method } & True & $J_{n}(k \rho)=0$ & $\mathrm{e}^{\mathrm{i} n \theta}$ & $\begin{array}{c}u_{n}(a, \phi)=\pi^{2} R J_{n}(k a) Y_{n}(k \rho) \cos (n \phi)^{*} \\
\bar{u}_{n}(a, \phi)=J_{n}(k a) \cos (n \phi)^{\dagger}\end{array}$ \\
\hline & Spurious & $Y_{n}(k \rho)=0$ & $\mathrm{e}^{\mathrm{i} n \theta}$ & $\begin{array}{c}u_{n}(a, \phi)=\pi^{2} R J_{n}(k a) Y_{n}(k \rho) \cos (n \phi)^{\ddagger} \\
\bar{u}_{n}(a, \phi)=J_{n}(k a) \cos (n \phi)^{\dagger}\end{array}$ \\
\hline \multirow[t]{2}{*}{ LM method } & True & $J_{n}(k \rho)=0$ & $\mathrm{e}^{\mathrm{i} n \theta}$ & $\begin{array}{c}u_{n}(a, \phi)=\pi^{2} R J_{n}(k a) Y_{n}(k \rho) \cos (n \phi)^{*} \\
\bar{u}_{n}(a, \phi)=J_{n}(k a) \cos (n \phi)^{\dagger}\end{array}$ \\
\hline & Spurious & $Y_{n}^{\prime}(k \rho)=0$ & $\mathrm{e}^{\mathrm{i} n \theta}$ & $\begin{array}{c}u_{n}(a, \phi)=\pi^{2} R J_{n}(k a) Y_{n}(k \rho) \cos (n \phi)^{\ddagger} \\
\bar{u}_{n}(a, \phi)=J_{n}(k a) \cos (n \phi)^{\dagger}\end{array}$ \\
\hline
\end{tabular}

*Non-trivial solution.

$\dagger$ Non-trivial solution after normalization.

¥Trivial solution without normalization.

Table IV. True and spurious systems for the Neumann problem using the real-part UT and LM BEMs.

\begin{tabular}{|c|c|c|c|c|}
\hline & \multicolumn{2}{|c|}{ Eigenequation } & $\begin{array}{l}\text { Eigenmode } \\
\text { (boundary) }\end{array}$ & $\begin{array}{l}\text { Eigenmode(interior): } u_{n}(a, \phi) \text { (unnormalized) } \\
\text { Eigenmode(interior): } \bar{u}_{n}(a, \phi) \text { (normalized) }\end{array}$ \\
\hline \multirow[t]{2}{*}{ UT method } & True & $J_{n}^{\prime}(k \rho)=0$ & $\mathrm{e}^{\mathrm{i} n \theta}$ & $\begin{array}{c}u_{n}(a, \phi)=\pi^{2} R J_{n}(k a) Y_{n}^{\prime}(k \rho) \cos (n \phi)^{*} \\
\bar{u}_{n}(a, \phi)=J_{n}(k a) \cos (n \phi)^{\dagger}\end{array}$ \\
\hline & Spurious & $Y_{n}(k \rho)=0$ & $\mathrm{e}^{\mathrm{i} n \theta}$ & $\begin{array}{c}u_{n}(a, \phi)=\pi^{2} R J_{n}(k a) Y_{n}^{\prime}(k \rho) \cos (n \phi)^{\ddagger} \\
\bar{u}_{n}(a, \phi)=J_{n}(k a) \cos (n \phi)^{\dagger}\end{array}$ \\
\hline \multirow[t]{2}{*}{ LM method } & True & $J_{n}^{\prime}(k \rho)=0$ & $\mathrm{e}^{\mathrm{i} n \theta}$ & $\begin{array}{c}u_{n}(a, \phi)=\pi^{2} R J_{n}(k a) Y_{n}^{\prime}(k \rho) \cos (n \phi)^{*} \\
\bar{u}_{n}(a, \phi)=J_{n}(k a) \cos (n \phi)^{\dagger}\end{array}$ \\
\hline & Spurious & $Y_{n}^{\prime}(k \rho)=0$ & $\mathrm{e}^{\mathrm{i} n \theta}$ & $\begin{array}{c}u_{n}(a, \phi)=\pi^{2} R J_{n}(k a) Y_{n}^{\prime}(k \rho) \cos (n \phi)^{\ddagger} \\
\bar{u}_{n}(a, \phi)=J_{n}(k a) \cos (n \phi)^{\dagger}\end{array}$ \\
\hline
\end{tabular}

*Non-trivial solution.

$\dagger$ Non-trivial solution after normalization.

$\ddagger$ Trivial solution without normalization.

Based on the solution in terms of double-layer potential, we have

$$
\begin{aligned}
u_{n}(a, \phi) & =\int_{0}^{2 \pi} \sum_{m=-\infty}^{\infty} \frac{k \pi}{2} J_{m}(k a) Y_{m}^{\prime}(k R) \cos (m(\theta-\phi)) \cos (n \theta) R \mathrm{~d} \theta \\
& =\pi^{2} R J_{n}(k a) Y_{n}^{\prime}(k R) \cos (n \phi), \quad 0<a<\rho, \quad 0<\phi<2 \pi
\end{aligned}
$$

Similarly, the true and spurious solutions for the eigenvalues and eigenfunctions can be easily derived using direct methods. The results are shown in Tables III and IV for the Dirichlet and Neumann problems, respectively. It is interesting to find that the UT method has the trivial 

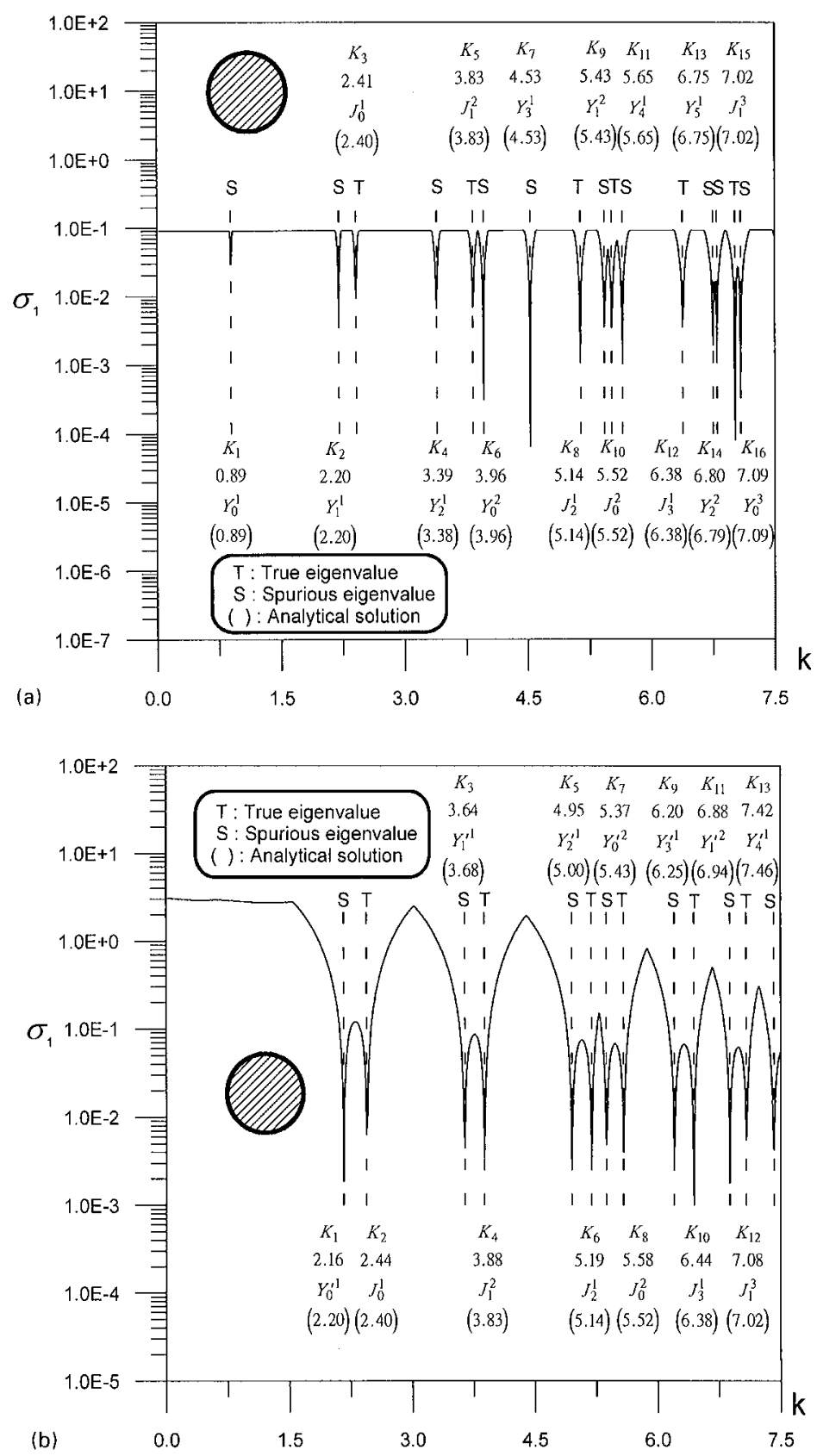

Figure 2. (a) The minimum singular values $\sigma_{1}$ versus $k$ using the real-part UT equation only for a circular cavity subject to Dirichlet boundary conditions; (b) the minimum singular values $\sigma_{1}$ versus $k$ using the real-part LM equation only for a circular cavity subject to Dirichlet boundary conditions; (c) the minimum singular values $\sigma_{1}$ versus $k$ using the real-part UT and LM equations for a circular cavity subject to Dirichlet boundary conditions; (d) the second minimum singular values $\sigma_{2}$ versus $k$ using the real-part UT and LM equations for a circular cavity subject to Dirichlet boundary conditions. 

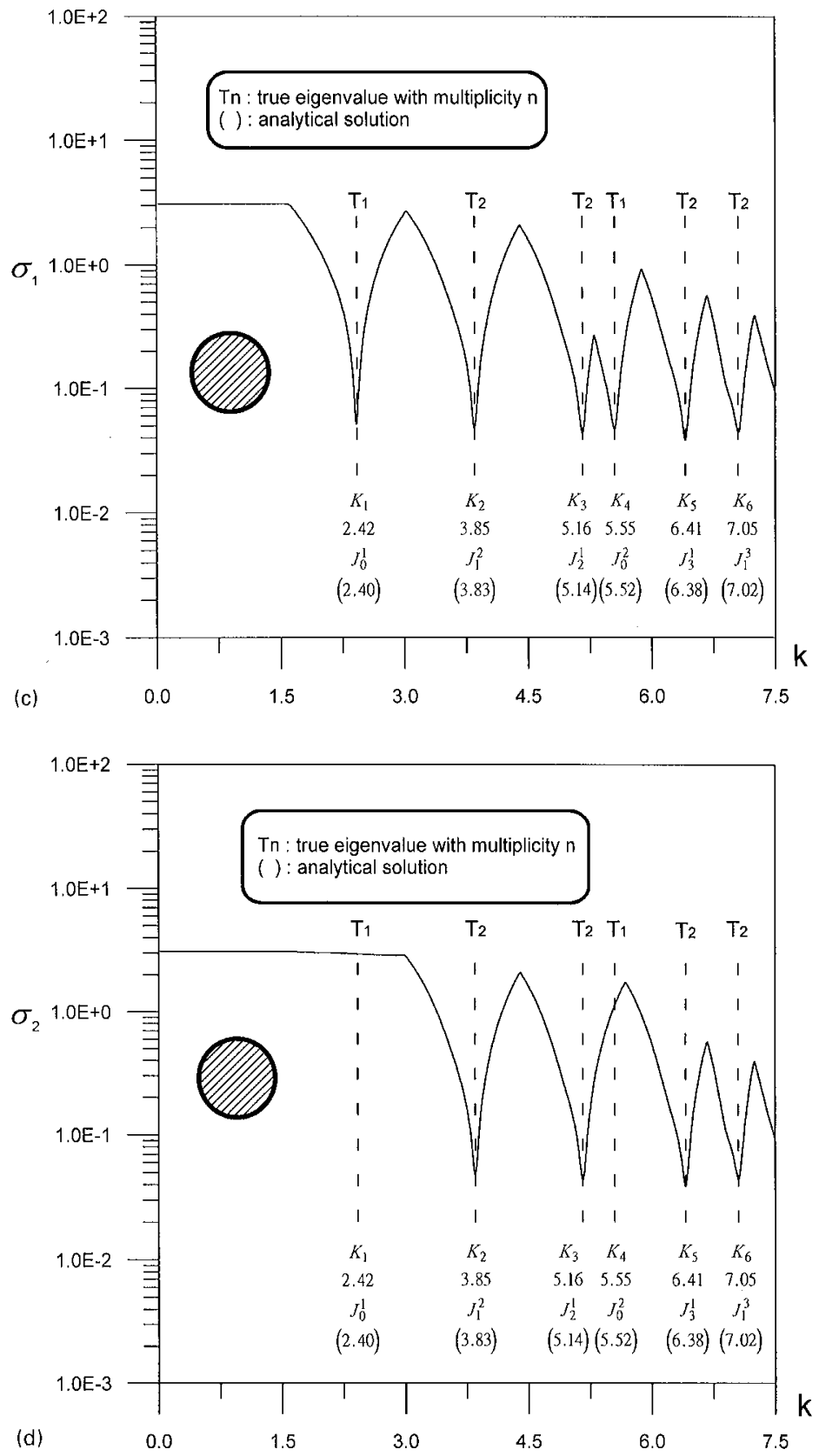

Figure 2. Continued. 

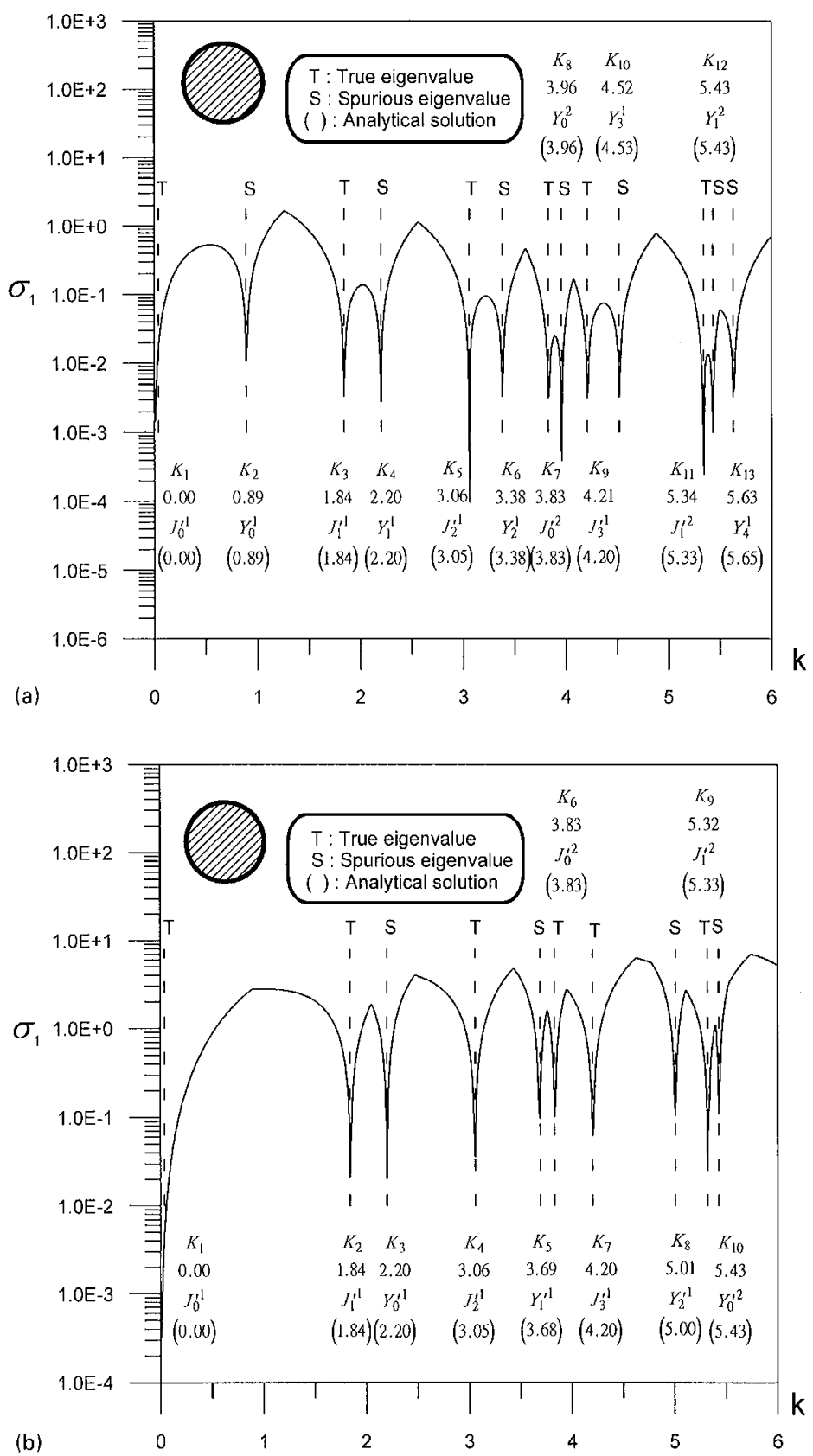

Figure 3. (a) The minimum singular values $\sigma_{1}$ versus $k$ using the real-part UT equation only for a circular cavity subject to Neumann boundary conditions; (b) the minimum singular values $\sigma_{1}$ versus $k$ using the real-part LM equation only for a circular cavity subject to Neumann boundary conditions; (c) the minimum singular values $\sigma_{1}$ versus $k$ using the real-part UT and LM equations for a circular cavity subject to Neumann boundary conditions; (d) the second minimum singular values $\sigma_{2}$ versus $k$ using the real-part UT and LM equations for a circular cavity subject to Neumann boundary conditions. 

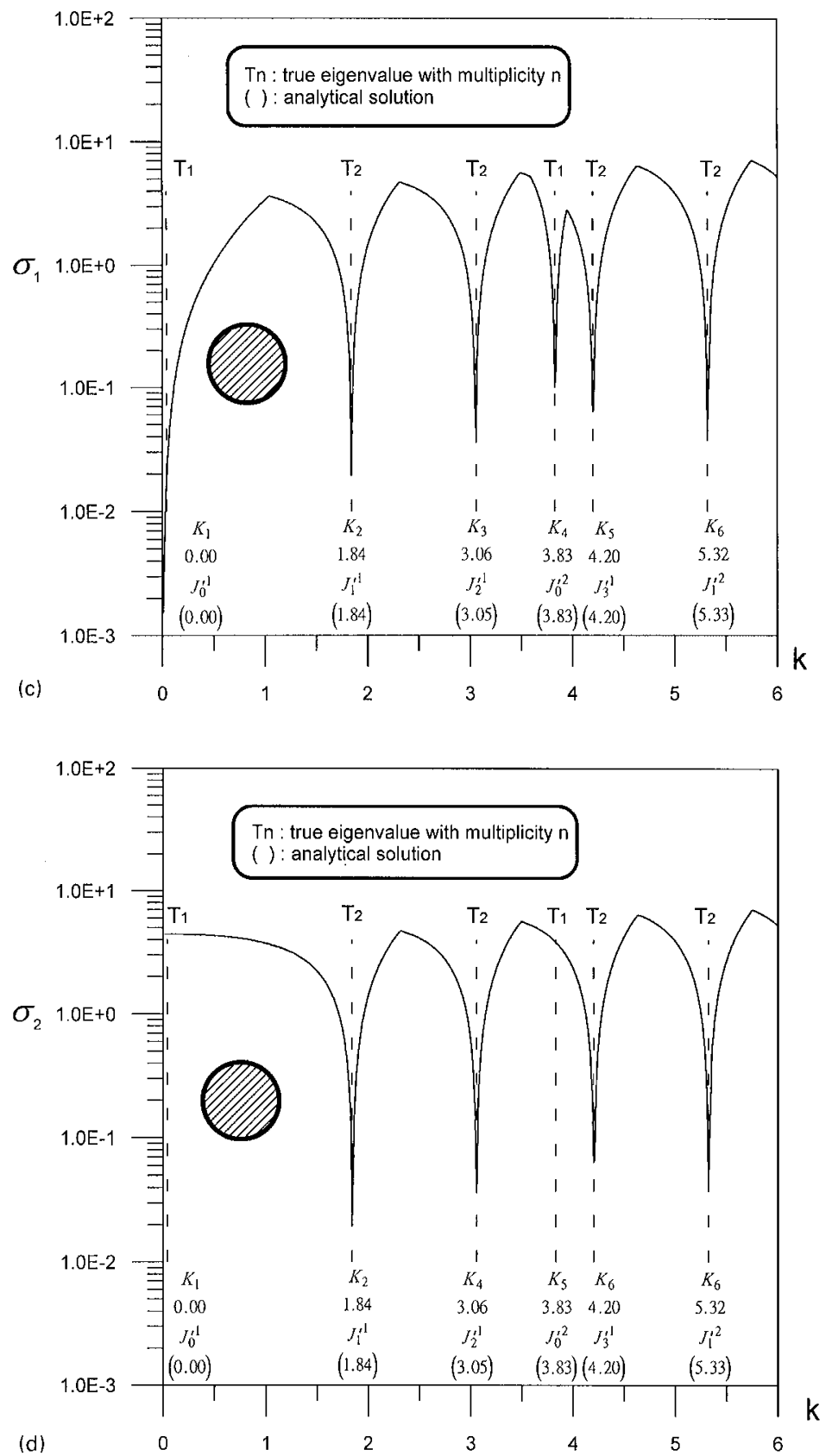

Figure 3. Continued. 

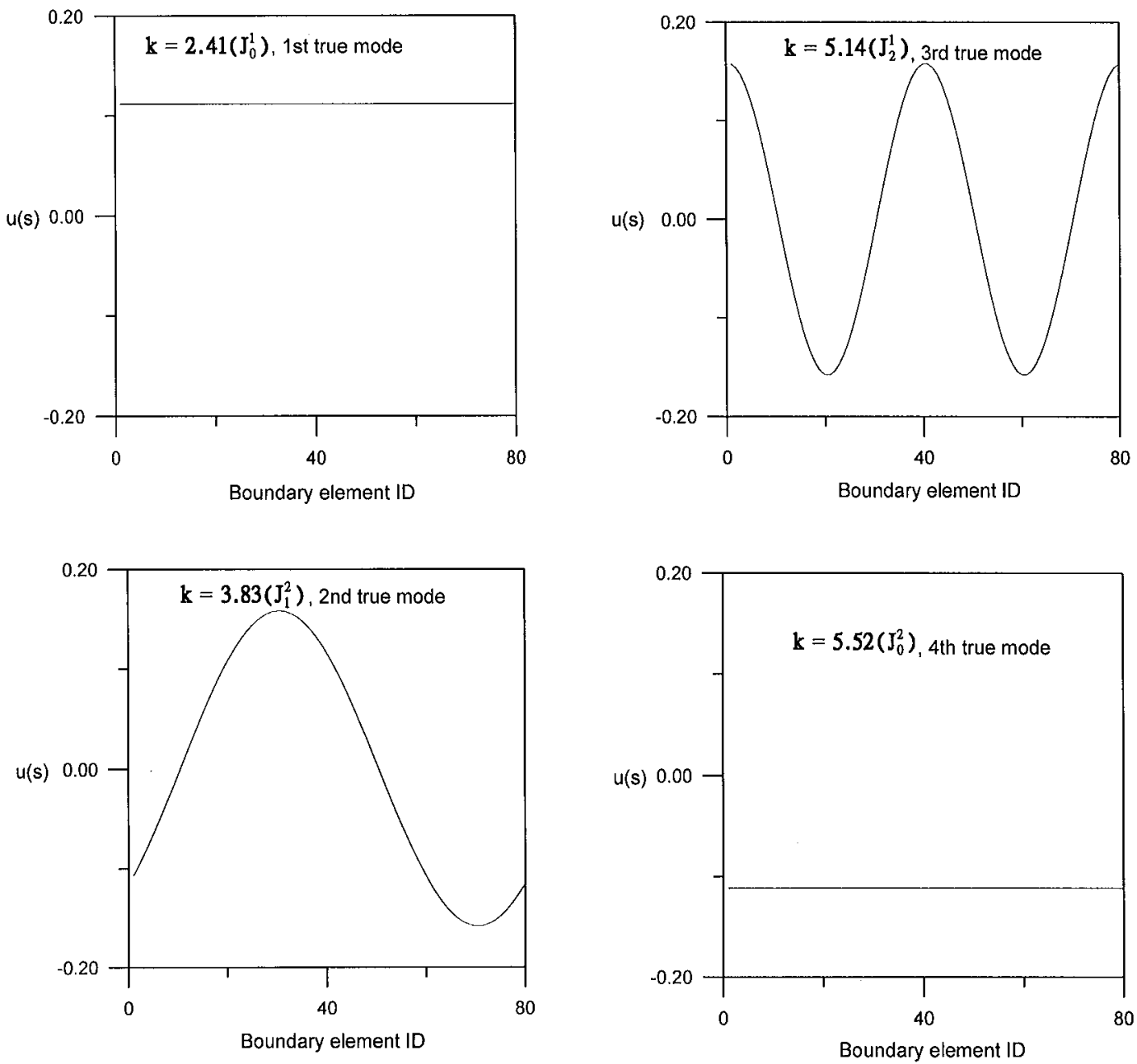

Figure 4. The first four true boundary eigenmodes for the Dirichlet problem.

eigensolution (since $Y_{n}(k \rho)$ is embedded in the solution) in the case of spurious eigenvalue where $k$ satisfies $Y_{n}(k \rho)=0$ as shown in Table III. The amplitude for the eigensolution is found to be very small but not zero numerically as shown in Figure 7 . The nodal line may mislead us to take it for granted that it is a true solution.

\section{NUMERICAL EXAMPLES}

Example 1. A circular cavity with a radius $(\rho=1 \mathrm{~m})$ subject to the Dirichlet boundary condition $(u=0, \rho=1)$. 

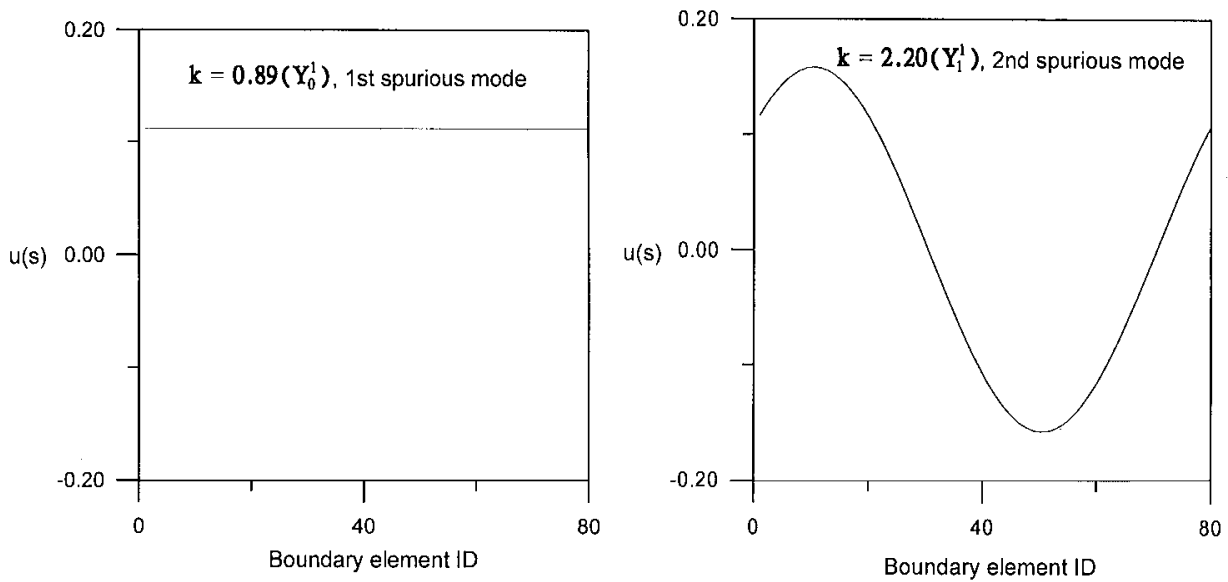

Figure 5. The first two spurious boundary eigenmodes for the Dirichlet problem.

In this case, an analytical solution is available as follows:

eigenequation: $J_{m}\left(k_{m n}\right)=0, m, n=0,1,2,3 \ldots$;

eigenmode: $u(a, \theta)=J_{m}\left(k_{m n} a\right) \mathrm{e}^{i m \theta}, 0<a<\rho, 0<\theta<2 \pi$.

Eighty elements are adopted in the boundary element mesh. Since two alternatives, the UT or LM equation, can be used to collocate on the boundary, two results from the UT and LM methods can be obtained. Figure 2(a) shows the minimum singular value versus $k$. The true eigenvalues contaminated by spurious eigenvalues can be obtained as shown in Figure 2(a) by considering the near zero minimum singular values if only the UT equation is chosen. The true eigenvalues occur at the positions of zeros for $J_{m}\left(k_{m n} \rho\right)$ while the spurious eigenvalues occur at the positions of zeros for $Y_{m}\left(k_{m n} \rho\right) . J_{m}^{n}$ and $Y_{m}^{n}$ in Figure 2(a) denote the roots for $k$ which satisfy $J_{m}\left(k_{m n} \rho\right)=0$ and $Y_{m}\left(k_{m n} \rho\right)=0$, respectively. In a similar way, the true eigenvalues contaminated by spurious eigenvalues can be obtained as shown in Figure 2(b) by considering the near zero minimum singular values if only the LM equation is chosen. The true eigenvalues occur at the positions of zeros for $J_{m}\left(k_{m n} \rho\right)$ while the spurious eigenvalues occur at the positions of zeros for $Y_{m}^{\prime}\left(k_{m n} \rho\right)$. $Y_{m}^{n^{\prime}}$ in Figire 2(b) denotes the roots for $k$ which satisfy $Y_{m}^{\prime}\left(k_{m n} \rho\right)=0$. It is interesting to find that no spurious eigenvalues occur as shown in Figure 2(c) when the UT and LM equations are combined. Also, the roots with multiplicity 2 are found in Figure 2(d), where both $\sigma_{1}$ and $\sigma_{2}$ approach zero at the same time.

Example 2. A circular cavity with a radius $(\rho=1 \mathrm{~m})$ subject to the Neumann boundary condition $(t=0, \rho=1)$.

In this case, an analytical solution is available as follows:

eigenequation: $J_{m}^{\prime}\left(k_{m n}\right)=0, m, n=0,1,2,3, \ldots$;

eigenmode: $u(a, \theta)=J_{m}\left(k_{m n} a\right) \mathrm{e}^{i m \theta}, 0<a<\rho, 0<\theta<2 \pi$.

Eighty elements are adopted in the boundary element mesh. Since two alternatives, the UT or LM equation, can be used to collocate on the boundary, two results from the UT and LM methods 
$\mathbf{k}=2.41\left(\mathrm{~J}_{0}^{1}\right), 1$ st true interior mode

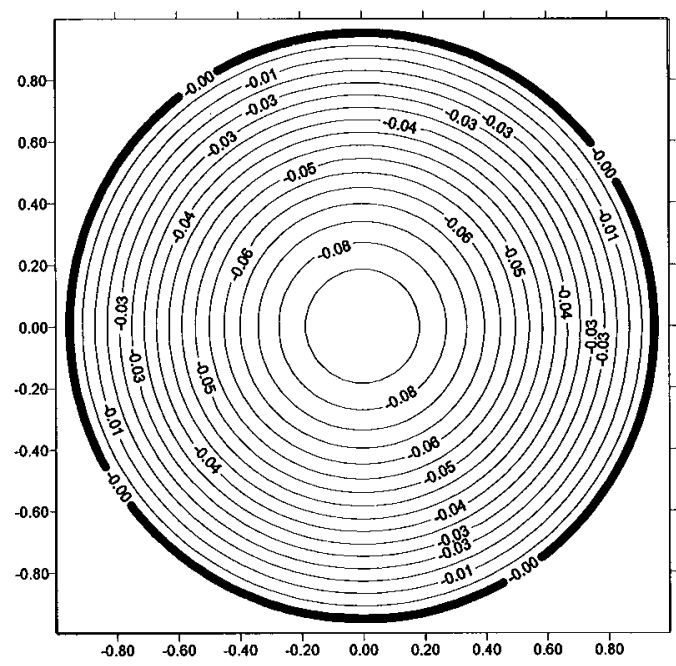

$\mathbf{k}=3.83\left(\mathrm{~J}_{1}^{2}\right)$, 2nd true interior mode

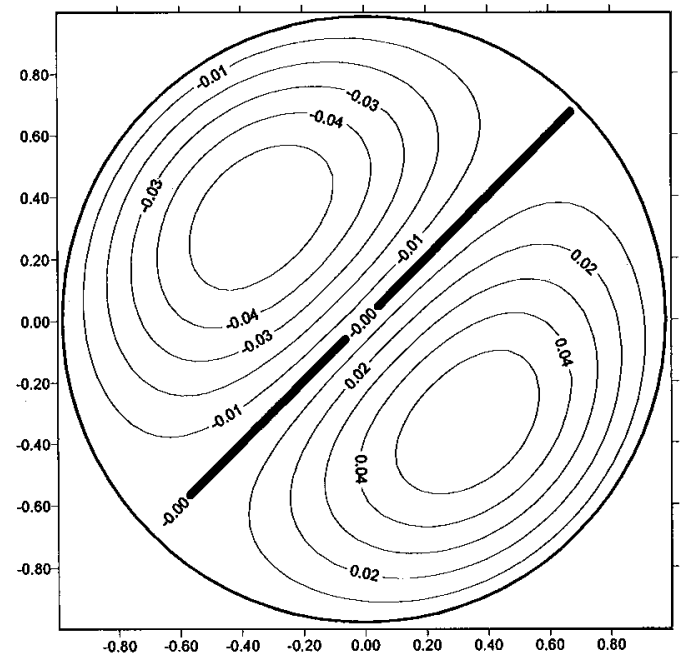

$\mathrm{k}=5.14\left(\mathrm{~J}_{2}^{1}\right)$, 3rd true interior mode

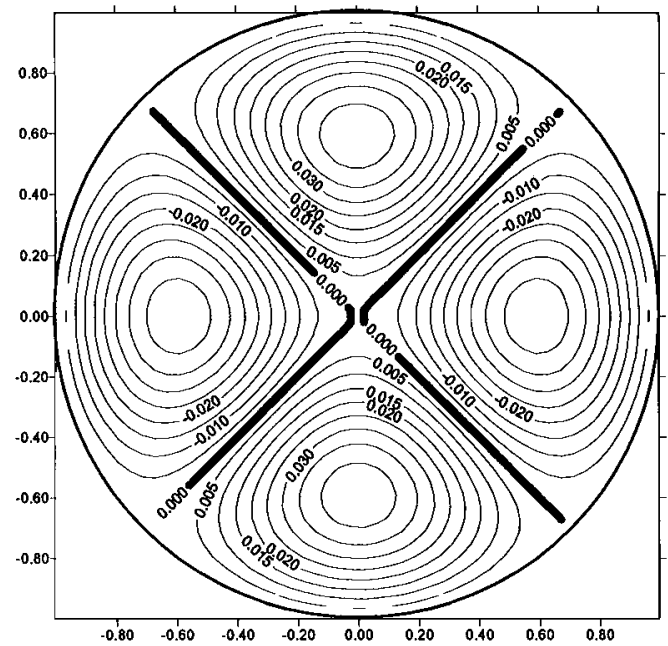

$\mathrm{k}=5.52\left(\mathrm{~J}_{0}^{2}\right)$, th true interior mode

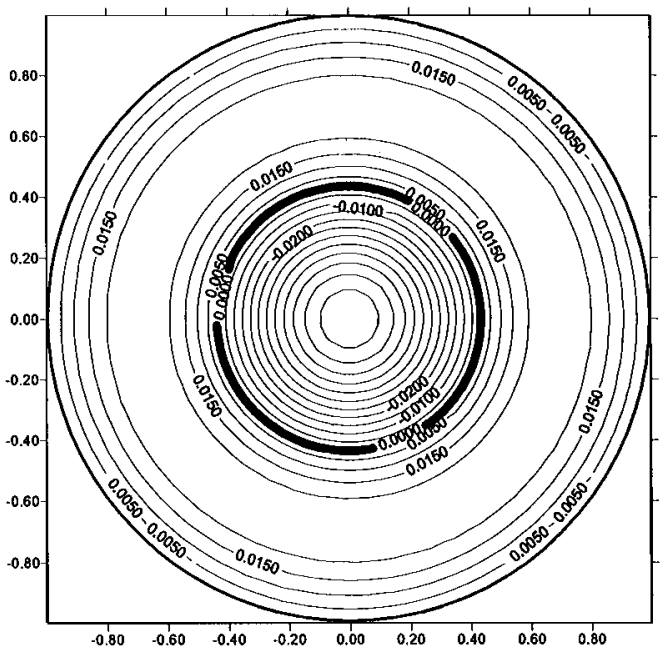

Figure 6. The first four true interior eigenmodes for the Dirichlet problem.

can be obtained. Figure 3(a) shows the minimum singular value versus $k$. The true eigenvalues contaminated by spurious eigenvalues can be obtained as shown in Figure 3(a) by considering the near zero minimum singular values if only the $U T$ equation is chosen. The true eigenvalues occur at the positions of zeros for $J_{m}^{\prime}\left(k_{m n} \rho\right)$ while the spurious eigenvalues occur at the positions of zeros for $Y_{m}\left(k_{m n} \rho\right)$. $J_{m}^{n^{\prime}}$ and $Y_{m}^{n}$ in Figure 3(a) denote the roots for $k$ which satisfy $J_{m}^{\prime}\left(k_{m n} \rho\right)=0$ 
$\mathrm{k}=0.89\left(\mathrm{Y}_{0}^{1}\right), 1$ st spurious interior mode
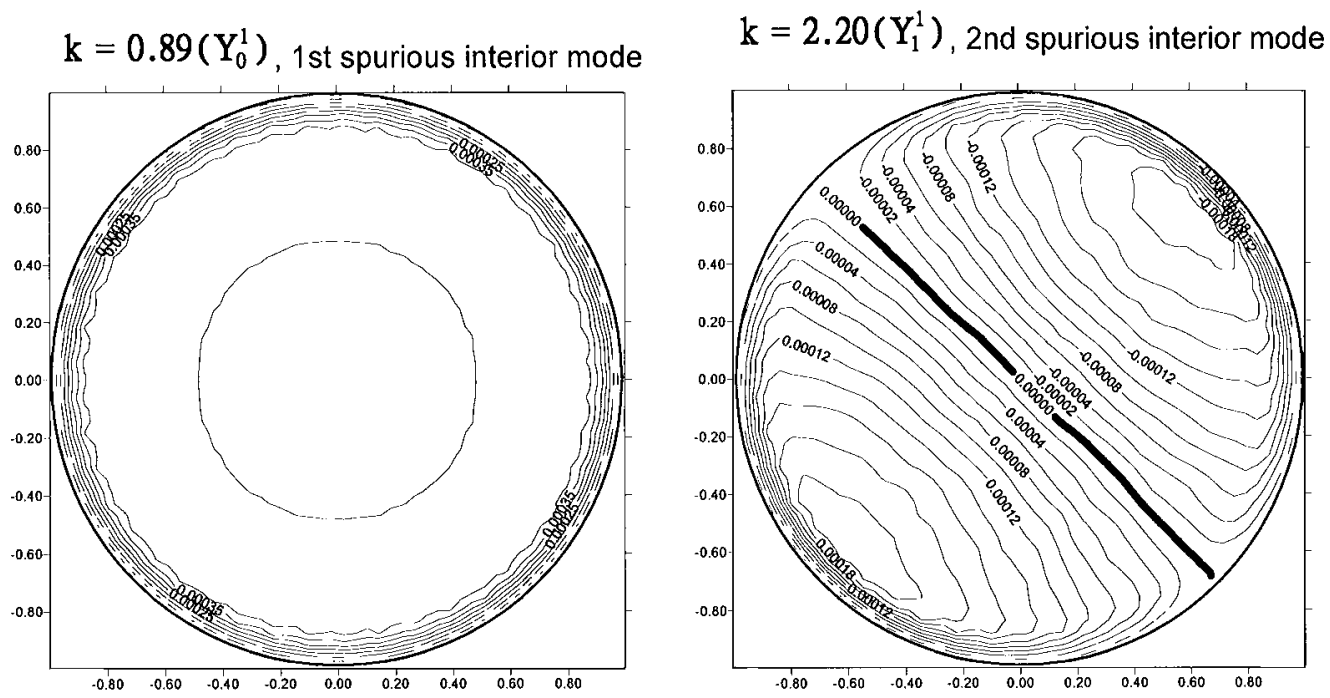

Figure 7. The first two spurious interior eigenmodes for the Dirichlet problem.

and $Y_{m}\left(k_{m n} \rho\right)=0$, respectively. In a similar way, the true eigenvalues contaminated by spurious eigenvalues can be obtained as shown in Figure 3(b) by considering the near zero minimum singular values if only the LM equation is chosen. The true eigenvalues occur at the positions of zeros for $J_{m}^{\prime}\left(k_{m n} \rho\right)$ while the spurious eigenvalues occur at the positions of zeros for $Y_{m}^{\prime}\left(k_{m n} \rho\right)$. $Y_{m}^{n^{\prime}}$ in Figure 3(b) denotes the roots for $k$ which satisfy $Y_{m}^{\prime}\left(k_{m n} \rho\right)=0$. It is interesting to find that no spurious eigenvalues occur as shown in Figure 3(c) when the UT and LM equations are combined. Also, the roots with multiplicity 2 are found in Figure 3(d), where both $\sigma_{1}$ and $\sigma_{2}$ approach zero at the same time. Good agreement for the two cases can be obtained. Also, the first four true and two spurious boundary eigenmodes are shown in Figures 4 and 5 for the Dirichlet problem, respectively. As derived in the analytical formulation, the nodal lines for the true and spurious interior modes are found to be the same as those shown in Figures 6 and 7, respectively. However, their eigenvalues are different.

\section{CONCLUSIONS}

The real-part dual BEM in conjunction with the SVD technique has been applied to determine the true and spurious eigensolutions of a circular cavity subject to the Dirichelet and Neumann boundary conditions. The spurious eigenvalues have been successfully predicted analytically and found numerically. The true eigenvalues obtained by the real-part dual BEM also match very well with the exact solutions. In addition, their multiplicities have been examined. The true and spurious eigenvalues, which occur at the positions of the zeros of the associated Bessel function for the spurious solutions were successfully filtered out using the SVD technique. Numerical experiments have been performed using the direct methods, and the results are satisfactory. 


\section{ACKNOWLEDGEMENTS}

Financial support from the National Science Council under Grants No. NSC-88-2211-E-019-005 and NSC88-2815-C-019-004-E for National Taiwan Ocean University is gratefully acknowledged.

\section{REFERENCES}

1. De Mey G. Calculation of the Helmholtz equation by an integral equation. International Journal for Numerical Methods in Engineering 1976; 10:59-66.

2. De Mey G. A simplified integral equation method for the calculation of the eigenvalues of Helmholtz equation. International Journal for Numerical Methods in Engineering 1977; 11:1340-1342.

3. Hutchinson JR. An alternative BEM formulation applied to membrane vibrations. In: Boundary Elements VII, Brebbia CA, Maier G. (eds). Springer: Berlin, 1985.

4. Tai GRG, Shaw RP. Helmholtz equation eigenvalues and eigenmodes for arbitrary domains. Journal of the Acoustical Society of America 1974; 56:796-804.

5. Chen JT. Recent development of dual BEM in acoustic problems, keynote lecture. Proceedings of the fourth World Congress on Computational Mechanics, Onate E, Idelsohn SR. (eds). Ceride Publications: Argentina, $1998 ; 106$.

6. Chen JT, Wong FC. Analytical derivations for one-dimensional eigenproblems using dual BEM and MRM. Engineering Analysis with Boundary Elements 1997; 20(1):25-33

7. Liou DY, Chen JT, Chen KH. A new method for determining the acoustic modes of a two-dimensional sound field. Journal of the Chinese Institute of Civil Hydraulics Engineering 1999; 11(2):299-310 (in Chinese).

8. Chen JT, Wong FC. Dual formulation of multiple reciprocity method for the acoustic mode of a cavity with a thin partition. Journal of Sound and Vibration 1998; 217(1):75-95.

9. Chen JT, Huang CS, Wong FC. Analysis and experiment for acoustic modes of a cavity containing an incomplete partition. Proceedings of the Fourth National Conference on Structural Engineering, vol. 1, 1998; 349-356.

10. Chen JT, Chen KH. Dual integral formulation for determining the acoustic modes of a two-dimensional cavity with a degenerate boundary. Engineering Analysis with Boundary Elements 1998; 21(2):105-116.

11. Chen JT, Hong H-K. Boundary Element Method, (2nd Edn). New World Press: Taipei, 1992 (in Chinese).

12. Chen KH, Chen JT. Liou DY. Dual boundary element analysis for an acoustic cavity with an incomplete partition. Chinese Journal of Mechanics 1998; 14(2):1-14 (in Chinese).

13. Chen JT, Chen KH, Chyuan SW. Numerical experiments for acoustic modes of a square cavity using dual BEM. Applied Acoustics 1999; 57(4):293-325.

14. Chen JT, Hong H-K. Review of dual integral representations with emphasis on hypersingular integrals and divergent series. Transactions of the ASME, Applied Mechanics Review 1999; 52(1):17-33.

15. Yeih W, Chen JT, Chen KH, Wong FC. A study on the multiple reciprocity method and complex-valued formulation for the Helmholtz equation. Advances in Engineering Software 1997; 29(1):7-12.

16. Yeih W, Chang YR, Chang CM, Chen JT. Applications of dual MRM for determining the natural frequencies and natural modes of a rod using the singular value decomposition method. Advances in Engineering Software 1999; 30(7):459-468.

17. Yeih W, Chen JT, Chang CM. Applications of dual MRM for determining the natural frequencies and natural modes of an Euler-Bernoulli beam using the singular value decomposition method. Engineering Analysis with Boundary Elements 1999; 23(4):339-360.

18. Chen JT, Huang CX, Chen KH. Determination of spurious eigenvalues and multiplicities of true eigenvalues using the real-part dual BEM. Computational Mechanics 1999; 24(1):41-51.

19. Chen JT, Liang MT, Chen, IL, Chyuan SW, Chen KH. Dual boundary element analysis of wave scattering from singularities. Wave Motion 1999; 30(4):367-381.

20. Kamiya N, Andoh E, Nogae K. A new complex-valued formulation and eigenvalue analysis of the Helmholtz equation by boundary element method. Advances in Engineering Software 1996; 26:219-227.

21. Nowak AJ, Neves AC. (eds). Multiple Reciprocity Boundary Element Method, Comp. Mech. Publ., Southampton, 1994.

22. Goldberg JL. Matrix Theory with Applications. McGraw-Hill: New York, 1991.

23. Press WT, Teukosky SA, Vetterling WT, Flannery BP. Numerical Recipes in FORTRAN (2nd edn). Cambridge University Press: New York, 1992.

24. Gloub GH, Van Loan CF. Matrix Computations (2nd edn). The Johns Hopkins University Press: Baltimore, 1989.

25. Chen JT, Kuo SR, Chen KH. A nonsingular integral formulation for the Helmholtz eigenproblems of a circular domain. Journal of the Chinese Institute of Engineers 1999; 22(6):729-739. 